\title{
Ultradiscrete Painlevé VI with Parity Variables
}

\author{
Kouichi TAKEMURA and Terumitsu TSUTSUI
}

Department of Mathematics, Faculty of Science and Technology, Chuo University, 1-13-27 Kasuga, Bunkyo-ku Tokyo 112-8551, Japan

E-mail: takemura@math.chuo-u.ac.jp

Received July 15, 2013, in final form November 11, 2013; Published online November 19, 2013

http://dx.doi.org/10.3842/SIGMA.2013.070

\begin{abstract}
We introduce a ultradiscretization with parity variables of the $q$-difference Painlevé VI system of equations. We show that ultradiscrete limit of Riccati-type solutions of $q$-Painlevé VI satisfies the ultradiscrete Painlevé VI system of equations with the parity variables, which is valid by using the parity variables. We study some solutions of the ultradiscrete Riccati-type equation and those of ultradiscrete Painlevé VI equation.
\end{abstract}

Key words: Painlevé equation; ultradiscrete; numerical solutions

2010 Mathematics Subject Classification: 39A13; 34M55; 37B15

\section{Introduction}

The Painlevé equations appear frequently in the problem of mathematical physics, and they have extremely rich structures of mathematics [1]. The $q$-Painlevé equations are $q$-difference analogues of the Painlevé equations [8], and most of them have symmetry of affine Weyl groups, which play important roles to analyze integrable systems $[6,10]$. On the other hand, cellular automaton has been studied actively and has been applied to vast areas of science and technology. Although some of cellular automaton describe complexity from their simple rule of evolution, some of them have integrability [12].

The ultradiscrete Painlevé equations are systems of cellular automaton and they are obtained by suitable limits (ultradiscretization) from the $q$-Painlevé equations [11]. We now explain ultradiscretization. We take positive variables $x, y, z$ and set $x=\exp (X / \varepsilon), y=\exp (Y / \varepsilon)$ and $z=\exp (Z / \varepsilon)$. If the variables $x, y, z$ satisfies $x+y=z$, then we have $\max (X, Y)=Z$ by the limit $\varepsilon \rightarrow+0$, which follows from the formula $\lim _{\varepsilon \rightarrow+0} \varepsilon \log (\exp (X / \varepsilon)+\exp (Y / \varepsilon))=\max (X, Y)$. It is easy to confirm that the relation $x y=z$ (resp. $x / y=z$ ) corresponds to $X+Y=Z$ (resp. $X-Y=Z$ ). This procedure is sometimes called ultradiscretization. The addition, the multiplication and the division correspond to taking the maximum, the addition and the subtraction by the ultradiscretization. However the subtraction $x-y$ is not well-behaved by the ultradiscretezation. Moreover if some values of $x, y, z$ are negative, then the procedure does not work well. To overcome these troubles, Satsuma and his collaborators [4] introduced the ultradiscretization with parity variables, and they obtained the ultradiscrete Painlevé II with parity variables [2]. Then the ultradiscrete Airy function with parity variables appears as a special solution of the ultradiscrete Painlevé II with parity variables [2].

The $q$-difference Painlevé VI system of equations was discovered by Jimbo and Sakai [3], and it is written as

$$
\frac{z(t) z(q t)}{b_{3} b_{4}}=\frac{\left(y(t)-t a_{1}\right)\left(y(t)-t a_{2}\right)}{\left(y(t)-a_{3}\right)\left(y(t)-a_{4}\right)}, \quad \frac{y(t) y(q t)}{a_{3} a_{4}}=\frac{\left(z(q t)-t b_{1}\right)\left(z(q t)-t b_{2}\right)}{\left(z(q t)-b_{3}\right)\left(z(q t)-b_{4}\right)}
$$

with the constraint $b_{1} b_{2} a_{3} a_{4}=q a_{1} a_{2} b_{3} b_{4}$. The original Painlevé VI equation is recovered by the limit $q \rightarrow 1$ (see [3]). 
The $q$-Painlevé VI system has Riccati-type solutions in the special cases [3]. Namely, if $b_{1} a_{3}=q a_{1} b_{3}, b_{2} a_{4}=a_{2} b_{4}$ and the functions $y(t)$ and $z(t)$ satisfy the following Riccati-type equation:

$$
z(q t)=b_{4} \frac{y(t)-t a_{2}}{y(t)-a_{4}}, \quad y(q t)=a_{3} \frac{z(q t)-t b_{1}}{z(q t)-b_{3}},
$$

then the functions $y(t)$ and $z(t)$ satisfy the $q$-Painlevé VI system. It is also known that the Riccati-type equation has solutions expressed by $q$-hypergeometric functions $[3,9]$.

In this article, we consider ultradiscretization of the $q$-Painlevé VI system with parity variables. For each value $m$ of the independent variable, we associate the signs $\mathfrak{y}_{m}, \mathfrak{z}_{m} \in\{ \pm 1\}$ and the amplitudes $Y_{m}, Z_{m} \in \mathbb{R}$. Define a parity function $S(\zeta)$ for a sign variable $\zeta$ by $S(1)=0$ and $S(-1)=-\infty$. We now introduce the ultradiscrete Painlevé VI system of equations with the variables $\left(\mathfrak{y}_{m}, Y_{m}\right)$ and $\left(\mathfrak{z}_{m}, Z_{m}\right)$ by

$$
\begin{aligned}
\max & {\left[\max \left(A_{1}, A_{2}\right)+m Q+Y_{m}+B_{3}+B_{4}+S\left(\mathfrak{y}_{m}\right),\right.} \\
& \max \left(2 Y_{m}, A_{3}+A_{4}\right)+Z_{m}+Z_{m+1}+S\left(\mathfrak{z}_{m} \mathfrak{z} m+1\right), \\
& \left.\max \left(A_{3}, A_{4}\right)+Y_{m}+Z_{m}+Z_{m+1}+S\left(-\mathfrak{y}_{m} \mathfrak{z} m \mathfrak{z} m+1\right)\right] \\
= & \max \left[\max \left(2 m Q+A_{1}+A_{2}, 2 Y_{m}\right)+B_{3}+B_{4},\right. \\
& \max \left(A_{1}, A_{2}\right)+m Q+Y_{m}+B_{3}+B_{4}+S\left(-\mathfrak{y}_{m}\right), \\
& \max \left(2 Y_{m}, A_{3}+A_{4}\right)+Z_{m}+Z_{m+1}+S(-\mathfrak{z} m \mathfrak{z} m+1), \\
& \left.\max \left(A_{3}, A_{4}\right)+Y_{m}+Z_{m}+Z_{m+1}+S\left(\mathfrak{y}_{m} \mathfrak{z} m \mathfrak{z} m+1\right)\right], \\
\max & {\left[\max \left(B_{1}, B_{2}\right)+m Q+Z_{m+1}+A_{3}+A_{4}+S(\mathfrak{z} m+1),\right.} \\
& \max \left(2 Z_{m+1}, B_{3}+B_{4}\right)+Y_{m}+Y_{m+1}+S\left(\mathfrak{y}_{m} \mathfrak{y}_{m+1}\right), \\
& \left.\max \left(B_{3}, B_{4}\right)+Y_{m}+Y_{m+1}+Z_{m+1}+S\left(-\mathfrak{y}_{m} \mathfrak{y}_{m+1} \mathfrak{z} m+1\right)\right] \\
= & \max \left[\max \left(2 m Q+B_{1}+B_{2}, 2 Z_{m+1}\right)+A_{3}+A_{4},\right. \\
& \max \left(B_{1}, B_{2}\right)+m Q+Z_{m+1}+A_{3}+A_{4}+S\left(-\mathfrak{z}_{m+1}\right), \\
& \max \left(2 Z_{m+1}, B_{3}+B_{4}\right)+Y_{m}+Y_{m+1}+S\left(-\mathfrak{y}_{m} \mathfrak{y}_{m+1}\right), \\
& \left.\max \left(B_{3}, B_{4}\right)+Y_{m}+Y_{m+1}+Z_{m+1}+S\left(\mathfrak{y}_{m} \mathfrak{y}_{m+1} \mathfrak{z}_{m+1}\right)\right],
\end{aligned}
$$

with the constraint

$$
B_{1}+B_{2}+A_{3}+A_{4}=Q+A_{1}+A_{2}+B_{3}+B_{4} .
$$

On equations (3), (4) we ignore the terms containing $S(\zeta)=-\infty$ in the maximum. Note that ultradiscretization of the $q$-Painlevé VI equation without parity variables was already introduced by Ormerod [7], and it is recovered by fixing the parity variables by $\mathfrak{y}_{m}=\mathfrak{z}_{m}=-1$. Equations (3), (4) are obtained by ultradiscretization of the $q$-Painlevé VI system under the condition $a_{i}>0$ and $b_{i}>0(i=1,2,3,4)$, where the condition is used in the process of obtaining the original Painlevé VI equation in [3], and we can also obtain the ultradiscrete Painlevé VI system of equations which admits the parities of the parameters (see equations (17), (18)). On the ultradiscrete Painlevé VI system of equations with parity variables, we have existence of the solution of the initial value problem, although the uniqueness does not hold true. We also ultradiscretize the Riccati-type equation with parity variables and show that any solutions of the ultradiscrete Riccati-type equation satisfy the ultradiscrete Painlevé VI system. Here the ultradiscretization with parity variables is essential because the ultradiscrete Riccati-type equation does not have any solutions in the case $\mathfrak{y}_{m}=\mathfrak{z}_{m}=-1$.

We try to study the solutions of the ultradiscrete Riccati-type equation and those of the ultradiscrete Painlevé VI equation. We give examples of solutions which are described by piecewiselinear functions. Based on numerical calculations, we present a conjecture that the solutions 
are expressed as linear functions if the independent variable $m$ is enough large (for details see Conjecture 1).

This paper is organized as follows. In Section 2, we consider ultradiscretization of the Riccatitype equation and that of the $q$-Painlevé VI equation. We establish existence of the solution of the initial value problem for the ultradiscrete Painlevé VI system of equations. In Section 3, we show that any solutions of the ultradiscrete Riccati-type equation also satisfy the ultradiscrete Painlevé VI equation. In Section 4, we investigate solutions of the ultradiscrete Riccati-type equation and those of the ultradiscrete Painlevé VI equation.

\section{Ultradiscretization}

\subsection{Ultradiscretization of the Riccati-type equation}

We consider ultradiscretization of the Riccati-type equation at first, because the expression is simpler than those of the $q$-Painlevé VI equation.

To obtain the ultradiscrete limit, we set

$$
\begin{aligned}
& t=q^{m}, \quad q=e^{Q / \varepsilon}, \quad a_{i}=e^{A_{i} / \varepsilon}, \quad b_{i}=e^{B_{i} / \varepsilon}, \quad i=1,2,3,4, \\
& y\left(q^{m}\right)=\left(s\left(\mathfrak{y}_{m}\right)-s\left(-\mathfrak{y}_{m}\right)\right) e^{Y_{m} / \varepsilon}, \quad z\left(q^{m}\right)=\left(s\left(\mathfrak{z}_{m}\right)-s\left(-\mathfrak{z}_{m}\right)\right) e^{Z_{m} / \varepsilon},
\end{aligned}
$$

and define the parity functions $s(\zeta)$ and $S(\zeta)$ by

$$
s(\zeta)=\left\{\begin{array}{ll}
1, & \zeta=+1, \\
0, & \zeta=-1,
\end{array} \quad S(\zeta)= \begin{cases}0, & \zeta=+1 \\
-\infty, & \zeta=-1\end{cases}\right.
$$

Then we have $s(\zeta)=e^{S(\zeta) / \varepsilon}$ for $\varepsilon>0$. We substitute equation (6) into the equation

$$
z(q t)\left(y(t)-a_{4}\right)=b_{4}\left(y(t)-t a_{2}\right),
$$

which is equivalent to first equation of (2), and transpose the terms to disappear the minus signs. Then we have

$$
\begin{aligned}
& s\left(\mathfrak{z}_{m+1}\right) e^{Z_{m+1} / \varepsilon} s\left(\mathfrak{y}_{m}\right) e^{Y_{m} / \varepsilon}+s\left(-\mathfrak{z}_{m+1}\right) e^{Z_{m+1} / \varepsilon}\left\{s\left(-\mathfrak{y}_{m}\right) e^{Y_{m} / \varepsilon}+e^{A_{4} / \varepsilon}\right\} \\
& \quad+e^{B_{4} / \varepsilon}\left\{s\left(-\mathfrak{y}_{m}\right) e^{Y_{m} / \varepsilon}+e^{\left(m Q+A_{2}\right) / \varepsilon}\right\}=e^{B_{4} / \varepsilon} s\left(\mathfrak{y}_{m}\right) e^{Y_{m} / \varepsilon} \\
& \quad+s\left(\mathfrak{z}_{m+1}\right) e^{Z_{m+1} / \varepsilon}\left\{s\left(-\mathfrak{y}_{m}\right) e^{Y_{m} / \varepsilon}+e^{A_{4} / \varepsilon}\right\}+s\left(-\mathfrak{z}_{m+1}\right) e^{Z_{m+1} / \varepsilon} s\left(\mathfrak{y}_{m}\right) e^{Y_{m} / \varepsilon} .
\end{aligned}
$$

By using the formula $s(\mathfrak{y}) s(\mathfrak{z})+s(-\mathfrak{y}) s(-\mathfrak{z})=s(\mathfrak{y} \mathfrak{z})$ and taking the limit $\varepsilon \rightarrow+0$, we have

$$
\begin{aligned}
\max & {\left[m Q+A_{2}+B_{4}, Z_{m+1}+A_{4}+S\left(-\mathfrak{z}_{m+1}\right),\right.} \\
& \left.Y_{m}+B_{4}+S\left(-\mathfrak{y}_{m}\right), Y_{m}+Z_{m+1}+S\left(\mathfrak{y}_{m} \mathfrak{z}_{m+1}\right)\right] \\
= & \max \left[Z_{m+1}+A_{4}+S\left(\mathfrak{z}_{m+1}\right), Y_{m}+B_{4}+S\left(\mathfrak{y}_{m}\right), Y_{m}+Z_{m+1}+S\left(-\mathfrak{y}_{m} \mathfrak{z}_{m+1}\right)\right] .
\end{aligned}
$$

It follows from the second equation of (2) that

$$
\begin{aligned}
\max & {\left[m Q+A_{3}+B_{1}, Y_{m+1}+B_{3}+S\left(-\mathfrak{y}_{m+1}\right),\right.} \\
& \left.Z_{m+1}+A_{3}+S(-\mathfrak{z} m+1), Y_{m+1}+Z_{m+1}+S\left(\mathfrak{y}_{m+1} \mathfrak{z} m+1\right)\right] \\
= & \max \left[Z_{m+1}+A_{3}+S\left(\mathfrak{z}_{m+1}\right), Y_{m+1}+B_{3}+S\left(\mathfrak{y}_{m+1}\right),\right. \\
& \left.Y_{m+1}+Z_{m+1}+S\left(-\mathfrak{y}_{m+1} \mathfrak{z}_{m+1}\right)\right] .
\end{aligned}
$$

We call equations $(7),(8)$ the ultradiscrete Riccati-type equation with parity variables. By the ultradiscrete limit, the conditions $b_{1} a_{3}=q a_{1} b_{3}, b_{2} a_{4}=a_{2} b_{4}$ correspond to $B_{1}+A_{3}=Q+A_{1}+B_{3}$ and $B_{2}+A_{4}=A_{2}+B_{4}$. 
We write the equations for the amplitude variables by fixing the parity variables. Equation (7) for each case is written as

$$
\begin{aligned}
& \mathfrak{z}_{m+1}=1, \quad \mathfrak{y}_{m}=1 \quad \Rightarrow \\
& \max \left(m Q+A_{2}+B_{4}, Y_{m}+Z_{m+1}\right)=\max \left(Z_{m+1}+A_{4}, Y_{m}+B_{4}\right), \\
& \mathfrak{z}_{m+1}=1, \quad \mathfrak{y}_{m}=-1 \quad \Rightarrow \\
& \max \left(m Q+A_{2}, Y_{m}\right)+B_{4}=Z_{m+1}+\max \left(Y_{m}, A_{4}\right), \\
& \mathfrak{z}_{m+1}=-1, \quad \mathfrak{y}_{m}=1 \quad \Rightarrow \\
& \max \left(m Q+A_{2}+B_{4}, Z_{m+1}+A_{4}\right)=Y_{m}+\max \left(Z_{m+1}, B_{4}\right), \\
& \mathfrak{z}_{m+1}=-1, \quad \mathfrak{y}_{m}=-1 \quad \Rightarrow \\
& -\infty=\max \left(m Q+A_{2}+B_{4}, Y_{m}+Z_{m+1}, Z_{m+1}+A_{4}, Y_{m}+B_{4}\right) .
\end{aligned}
$$

There is no solution in the case $\mathfrak{z}_{m+1}=\mathfrak{y}_{m}=-1$. Equation (8) for each case is written as

$$
\begin{aligned}
& \mathfrak{z}_{m+1}=1, \quad \mathfrak{y}_{m+1}=1 \quad \Rightarrow \\
& \max \left(m Q+A_{3}+B_{1}, Y_{m+1}+Z_{m+1}\right)=\max \left(Z_{m+1}+A_{3}, Y_{m+1}+B_{3}\right), \\
& \mathfrak{z}_{m+1}=1, \quad \mathfrak{y}_{m+1}=-1 \quad \Rightarrow \\
& \max \left(m Q+A_{3}+B_{1}, Y_{m+1}+B_{3}\right)=Z_{m+1}+\max \left(Y_{m+1}, A_{3}\right), \\
& \mathfrak{z}_{m+1}=-1, \quad \mathfrak{y}_{m+1}=1 \quad \Rightarrow \\
& \max \left(m Q+B_{1}, Z_{m+1}\right)+A_{3}=Y_{m+1}+\max \left(Z_{m+1}, B_{3}\right), \\
& \mathfrak{z}_{m+1}=-1, \quad \mathfrak{y}_{m+1}=-1 \quad \Rightarrow \\
& -\infty=\max \left(m Q+A_{3}+B_{1}, Y_{m+1}+Z_{m+1}, Z_{m+1}+A_{3}, Y_{m+1}+B_{3}\right) .
\end{aligned}
$$

There is no solution in the case $\mathfrak{z}_{m+1}=\mathfrak{y}_{m+1}=-1$.

\section{$2.2 \quad$ Ultradiscretization of the $q$-Painlevé VI equation}

We can obtain similarly the ultradiscrete limit with parity variables of the $q$-Painlevé VI equation. We take a limit of equation (1) as $\varepsilon \rightarrow+0$ by setting the values as equation (6). By using the formulae

$$
\begin{aligned}
& s(\mathfrak{y})+s(-\mathfrak{y})=1, \quad s(\mathfrak{y}) s(-\mathfrak{y})=0, \\
& s(\mathfrak{y})^{2}=s(\mathfrak{y}), \quad s(\mathfrak{y}) s(\mathfrak{z})+s(-\mathfrak{y}) s(-\mathfrak{z})=s(\mathfrak{y} \mathfrak{z}), \\
& s(\mathfrak{y}) s(\mathfrak{z}) s(\mathfrak{w})+s(-\mathfrak{y}) s(-\mathfrak{z}) s(\mathfrak{w})+s(\mathfrak{y}) s(-\mathfrak{z}) s(-\mathfrak{w})+s(-\mathfrak{y}) s(\mathfrak{z}) s(-\mathfrak{w})=s(\mathfrak{y} \mathfrak{z}),
\end{aligned}
$$

we obtain the ultradiscrete Painlevé VI equation with parity variables (i.e. equations (3), (4)). In [13], another form of equations (3), (4) is derived with the details. By the ultradiscrete limit, the constraint $b_{1} b_{2} a_{3} a_{4}=q a_{1} a_{2} b_{3} b_{4}$ of the $q$-Painlevé VI system corresponds to equation (5).

We write the equations for the amplitude variables by fixing the parity variables. Equation (3) for each case is written as

$$
\begin{aligned}
& \mathfrak{y}_{m}=1, \quad \mathfrak{z} m \mathfrak{z}_{m+1}=1 \quad \Rightarrow \\
& \max \left(\max \left(2 Y_{m}, A_{3}+A_{4}\right)+Z_{m}+Z_{m+1}, \max \left(A_{1}, A_{2}\right)+m Q+Y_{m}+B_{3}+B_{4}\right) \\
& =\max \left(\max \left(2 m Q+A_{1}+A_{2}, 2 Y_{m}\right)+B_{3}+B_{4}, \max \left(A_{3}, A_{4}\right)+Y_{m}+Z_{m}+Z_{m+1}\right), \\
& \mathfrak{y}_{m}=1, \quad \mathfrak{z} m \mathfrak{z} m+1=-1 \quad \Rightarrow \\
& \max \left(\max \left(A_{3}, A_{4}\right)+Y_{m}+Z_{m}+Z_{m+1}, \max \left(A_{1}, A_{2}\right)+m Q+Y_{m}+B_{3}+B_{4}\right) \\
& =\max \left(\max \left(2 m Q+A_{1}+A_{2}, 2 Y_{m}\right)+B_{3}+B_{4}, \max \left(2 Y_{m}, A_{3}+A_{4}\right)+Z_{m}+Z_{m+1}\right),
\end{aligned}
$$




$$
\begin{aligned}
& \mathfrak{y}_{m}=-1, \quad \mathfrak{z} m \mathfrak{z}_{m+1}=1 \quad \Rightarrow \\
& Z_{m}+Z_{m+1}+\max \left(A_{3}, Y_{m}\right)+\max \left(A_{4}, Y_{m}\right) \\
& \quad=B_{3}+B_{4}+\max \left(m Q+A_{1}, Y_{m}\right)+\max \left(m Q+A_{2}, Y_{m}\right), \\
& \mathfrak{y}_{m}=-1, \quad \mathfrak{z} m \mathfrak{z} m+1=-1 \Rightarrow \\
& -\infty=\max \left(\max \left(2 Y_{m}, A_{3}+A_{4}\right)+Z_{m}+Z_{m+1}, \max \left(A_{1}, A_{2}\right)+m Q+Y_{m}+B_{3}+B_{4},\right. \\
& \left.\left.\quad \max \left(2 m Q+A_{1}+A_{2}, 2 Y_{m}\right)+B_{3}+B_{4}, \max \left(A_{3}, A_{4}\right)+Y_{m}+Z_{m}+Z_{m+1}\right)\right) .
\end{aligned}
$$

In the case $\mathfrak{y}_{m}=-1, \mathfrak{z}_{m} \mathfrak{z}_{m+1}=1$, we used the associativity of the maximum and the addition, i.e.

$$
\max \left(X_{1}+W_{1}, X_{2}+W_{1}, X_{1}+W_{2}, X_{2}+W_{2}\right)=\max \left(X_{1}, X_{2}\right)+\max \left(W_{1}, W_{2}\right) .
$$

There is no solution in the case $\mathfrak{y}_{m}=\mathfrak{z} m \mathfrak{z} m+1=-1$. On the other hand, equation (4) for each case is written as

$$
\begin{aligned}
& \mathfrak{z} m+1=1, \quad \mathfrak{y}_{m} \mathfrak{y}_{m+1}=1 \quad \Rightarrow \\
& \max \left(\max \left(2 Z_{m+1}, B_{3}+B_{4}\right)+Y_{m}+Y_{m+1}, \max \left(B_{1}, B_{2}\right)+m Q+Z_{m+1}+A_{3}+A_{4}\right) \\
& =\max \left(\max \left(2 m Q+B_{1}+B_{2}, 2 Z_{m+1}\right)+A_{3}+A_{4}, \max \left(B_{3}, B_{4}\right)+Y_{m}+Y_{m+1}+Z_{m+1}\right), \\
& \mathfrak{z} m+1=1, \quad \mathfrak{y}_{m} \mathfrak{y}_{m+1}=-1 \quad \Rightarrow \\
& \max \left(\max \left(B_{3}, B_{4}\right)+Y_{m}+Y_{m+1}+Z_{m+1}, \max \left(B_{1}, B_{2}\right)+m Q+A_{3}+A_{4}+Z_{m+1}\right) \\
& =\max \left(\max \left(2 m Q+B_{1}+B_{2}, 2 Z_{m+1}\right)+A_{3}+A_{4}, \max \left(2 Z_{m+1}, B_{3}+B_{4}\right)+Y_{m}+Y_{m+1}\right), \\
& \mathfrak{z} m+1=-1, \quad \mathfrak{y}_{m} \mathfrak{y}_{m+1}=1 \quad \Rightarrow \\
& Y_{m}+Y_{m+1}+\max \left(B_{3}, Z_{m+1}\right)+\max \left(B_{4}, Z_{m+1}\right) \\
& =A_{3}+A_{4}+\max \left(m Q+B_{1}, Z_{m+1}\right)+\max \left(m Q+B_{2}, Z_{m+1}\right), \\
& \mathfrak{z} m+1=-1, \quad \mathfrak{y}_{m} \mathfrak{y}_{m+1}=-1 \quad \Rightarrow \\
& -\infty=\max \left(\max \left(2 Z_{m+1}, B_{3}+B_{4}\right)+Y_{m}+Y_{m+1}, \max \left(B_{1}, B_{2}\right)+m Q+Z_{m+1}+A_{3}+A_{4},\right. \\
& \left.\left.\max \left(2 m Q+B_{1}+B_{2}, 2 Z_{m+1}\right)+A_{3}+A_{4}, \max \left(B_{3}, B_{4}\right)+Y_{m}+Y_{m+1}+Z_{m+1}\right)\right) .
\end{aligned}
$$

There is no solution in the case $\mathfrak{z}_{m+1}=\mathfrak{y}_{m} \mathfrak{y}_{m+1}=-1$.

We can also obtain the ultradiscrete Painlevé VI system of equations which admits the parities of parameters. Set

$$
a_{i}=\left(s\left(\mathfrak{a}_{i}\right)-s\left(-\mathfrak{a}_{i}\right)\right) e^{A_{i} / \varepsilon}, \quad b_{i}=\left(s\left(\mathfrak{b}_{i}\right)-s\left(-\mathfrak{b}_{i}\right)\right) e^{B_{i} / \varepsilon}, \quad i=1,2,3,4,
$$

in addition to equation (6), where $\mathfrak{a}_{i}, \mathfrak{b}_{i} \in\{ \pm 1\}$ represent the signs of $a_{i}, b_{i}(i=1,2,3,4)$. We take the ultradiscrete limit $(\varepsilon \rightarrow+0)$. Then we have

$$
\begin{aligned}
\max & {\left[2 m Q+A_{1}+A_{2}+B_{3}+B_{4}+S\left(-\mathfrak{a}_{1} \mathfrak{a}_{2} \mathfrak{b}_{3} \mathfrak{b}_{4}\right),\right.} \\
& 2 Y_{m}+B_{3}+B_{4}+S\left(-\mathfrak{b}_{3} \mathfrak{b}_{4}\right), \\
& Y_{m}+m Q+A_{1}+B_{3}+B_{4}+S\left(\mathfrak{a}_{1} \mathfrak{b}_{3} \mathfrak{b}_{4} \mathfrak{y}_{m}\right), \\
& Y_{m}+m Q+A_{2}+B_{3}+B_{4}+S\left(\mathfrak{a}_{2} \mathfrak{b}_{3} \mathfrak{b}_{4} \mathfrak{y}_{m}\right), \\
& 2 Y_{m}+Z_{m}+Z_{m+1}+S(\mathfrak{z} m \mathfrak{z} m+1) \\
& Z_{m}+Z_{m+1}+A_{3}+A_{4}+S\left(\mathfrak{a}_{3} \mathfrak{a}_{4} \mathfrak{z} m \mathfrak{z} m+1\right), \\
& Y_{m}+Z_{m}+Z_{m+1}+A_{3}+S\left(-\mathfrak{a}_{3} \mathfrak{y}_{m} \mathfrak{z} m \mathfrak{z} m+1\right), \\
& \left.Y_{m}+Z_{m}+Z_{m+1}+A_{4}+S\left(-\mathfrak{a}_{4} \mathfrak{y}_{m} \mathfrak{z} m \mathfrak{z} m+1\right)\right] \\
= & \max \left[2 m Q+A_{1}+A_{2}+B_{3}+B_{4}+S\left(\mathfrak{a}_{1} \mathfrak{a}_{2} \mathfrak{b}_{3} \mathfrak{b}_{4}\right),\right. \\
& 2 Y_{m}+B_{3}+B_{4}+S\left(\mathfrak{b}_{3} \mathfrak{b}_{4}\right), \\
& Y_{m}+m Q+A_{1}+B_{3}+B_{4}+S\left(-\mathfrak{a}_{1} \mathfrak{b}_{3} \mathfrak{b}_{4} \mathfrak{y}_{m}\right),
\end{aligned}
$$




$$
\begin{aligned}
& Y_{m}+m Q+A_{2}+B_{3}+B_{4}+S\left(-\mathfrak{a}_{2} \mathfrak{b}_{3} \mathfrak{b}_{4} \mathfrak{y}_{m}\right), \\
& 2 Y_{m}+Z_{m}+Z_{m+1}+S(-\mathfrak{z} m \mathfrak{z} m+1), \\
& Z_{m}+Z_{m+1}+A_{3}+A_{4}+S\left(-\mathfrak{a}_{3} \mathfrak{a}_{4} \mathfrak{z}_{m} \mathfrak{z}_{m+1}\right), \\
& Y_{m}+Z_{m}+Z_{m+1}+A_{3}+S\left(\mathfrak{a}_{3} \mathfrak{y}_{m} \mathfrak{z}_{m} \mathfrak{z}_{m+1}\right), \\
& \left.Y_{m}+Z_{m}+Z_{m+1}+A_{4}+S\left(\mathfrak{a}_{4} \mathfrak{y}_{m} \mathfrak{z} m \mathfrak{z}_{m+1}\right)\right], \\
\max & {\left[2 m Q+A_{3}+A_{4}+B_{1}+B_{2}+S\left(-\mathfrak{a}_{3} \mathfrak{a}_{4} \mathfrak{b}_{1} \mathfrak{b}_{2}\right),\right.} \\
& 2 Z_{m+1}+A_{3}+A_{4}+S\left(-\mathfrak{a}_{3} \mathfrak{a}_{4}\right), \\
& Z_{m+1}+m Q+A_{3}+A_{4}+B_{1}+S\left(\mathfrak{a}_{3} \mathfrak{a}_{4} \mathfrak{b}_{1} \mathfrak{z}_{m+1}\right), \\
& Z_{m+1}+m Q+A_{3}+A_{4}+B_{2}+S\left(\mathfrak{a}_{3} \mathfrak{a}_{4} \mathfrak{b}_{2} \mathfrak{z} m+1\right), \\
& 2 Z_{m+1}+Y_{m}+Y_{m+1}+S\left(\mathfrak{y}_{m} \mathfrak{y}_{m+1}\right), \\
& Y_{m}+Y_{m+1}+B_{3}+B_{4}+S\left(\mathfrak{b}_{3} \mathfrak{b}_{4} \mathfrak{y}_{m} \mathfrak{y}_{m+1}\right), \\
& Y_{m}+Y_{m+1}+Z_{m+1}+B_{3}+S\left(-\mathfrak{b}_{3} \mathfrak{y}_{m} \mathfrak{y}_{m+1} \mathfrak{z} m+1\right), \\
& \left.Y_{m}+Y_{m+1}+Z_{m+1}+B_{4}+S\left(-\mathfrak{b}_{4} \mathfrak{y}_{m} \mathfrak{y}_{m+1} \mathfrak{z}_{m+1}\right)\right] \\
= & m a x\left[2 m Q+A_{3}+A_{4}+B_{1}+B_{2}+S\left(\mathfrak{a}_{3} \mathfrak{a}_{4} \mathfrak{b}_{1} \mathfrak{b}_{2}\right),\right. \\
& 2 Z_{m+1}+A_{3}+A_{4}+S\left(\mathfrak{a}_{3} \mathfrak{a}_{4}\right), \\
& Z_{m+1}+m Q+A_{3}+A_{4}+B_{1}+S\left(-\mathfrak{a}_{3} \mathfrak{a}_{4} \mathfrak{b}_{1 \mathfrak{z}} \mathfrak{z}_{m+1}\right), \\
& Z_{m+1}+m Q+A_{3}+A_{4}+B_{2}+S\left(-\mathfrak{a}_{3} \mathfrak{a}_{4} \mathfrak{b}_{2} \mathfrak{z}_{m+1}\right), \\
& 2 Z_{m+1}+Y_{m}+Y_{m+1}+S\left(-\mathfrak{y}_{m} \mathfrak{y}_{m+1}\right), \\
& Y_{m}+Y_{m+1}+B_{3}+B_{4}+S\left(-\mathfrak{b}_{3} \mathfrak{b}_{4} \mathfrak{y}_{m} \mathfrak{y}_{m+1}\right), \\
& Y_{m}+Y_{m+1}+Z_{m+1}+B_{3}+S\left(\mathfrak{b}_{3} \mathfrak{y}_{m} \mathfrak{y}_{m+1} \mathfrak{z}_{m+1}\right), \\
& \left.Y_{m}+Y_{m+1}+Z_{m+1}+B_{4}+S\left(\mathfrak{b}_{4} \mathfrak{y}_{m} \mathfrak{y}_{m+1} \mathfrak{z}_{m+1}\right)\right],
\end{aligned}
$$

with the constraint

$$
B_{1}+B_{2}+A_{3}+A_{4}=Q+A_{1}+A_{2}+B_{3}+B_{4}, \quad \mathfrak{a}_{1} \mathfrak{a}_{2} \mathfrak{a}_{3} \mathfrak{a}_{4}=\mathfrak{b}_{1} \mathfrak{b}_{2} \mathfrak{b}_{3} \mathfrak{b}_{4}
$$

By setting $\mathfrak{a}_{i}=\mathfrak{b}_{i}=+1(i=1,2,3,4)$, we recover equations (3), (4). In the rest of the paper, we consider the case $\mathfrak{a}_{i}=\mathfrak{b}_{i}=+1(i=1,2,3,4)$, i.e. equations (3), (4) for simplicity.

The ultradiscrete Painlevé VI equation with parity variables has solutions for any give initial values. Namely we have the following proposition:

Proposition 1. Let $n_{o} \in \mathbb{Z}, \tilde{\mathfrak{y}}_{o}, \tilde{\mathfrak{z}}_{o} \in\{ \pm 1\}$ and $y_{o}, z_{o} \in \mathbb{R}$. Then there exists a solution $\left(\mathfrak{y}_{n}, Y_{n}\right)$, $\left(\mathfrak{z}_{n}, Z_{n}\right)(n \in \mathbb{Z})$ of equations (3), (4) which satisfies the condition $\left(\mathfrak{y}_{n_{o}}, Y_{n_{o}}\right)=\left(\tilde{\mathfrak{y}}_{o}, y_{o}\right)$ and $\left(\mathfrak{z}_{n_{o}}, Z_{n_{o}}\right)=\left(\tilde{\mathfrak{z}}_{o}, z_{o}\right)$.

Proof. We show that, if the values $\left(\mathfrak{z}_{m}, Z_{m}\right)$ and $\left(\mathfrak{y}_{m}, Y_{m}\right)$ are fixed, then there exists $(\mathfrak{z} m+1$, $Z_{m+1}$ ) such that equation (3) is satisfied.

If $\mathfrak{y}_{m}=-1$, then we have $\mathfrak{z}_{m+1}=\mathfrak{z}_{m}$ and $Z_{m+1}$ is determined by equation (13).

Assume that $\mathfrak{y}_{m}=1$. Write $U=\max \left(2 Y_{m}, A_{3}+A_{4}\right), U^{\prime}=\max \left(A_{3}, A_{4}\right)+Y_{m}, V=$ $\max \left(2 m Q+A_{1}+A_{2}, 2 Y_{m}\right)$ and $V^{\prime}=\max \left(A_{1}, A_{2}\right)+m Q+Y_{m}$. If $U \geq U^{\prime}$ and $V \geq V^{\prime}$ (resp. $U<U^{\prime}$ and $V<V^{\prime}$ ), then equation (3) is satisfied by setting $\mathfrak{z}_{m+1}=\mathfrak{z}_{m}$ and $Z_{m+1}=$ $V-U+B_{3}+B_{4}-Z_{m}$ (resp. $Z_{m+1}=V^{\prime}-U^{\prime}+B_{3}+B_{4}-Z_{m}$ ) (see equation (11)). If $U \geq U^{\prime}$ and $V<V^{\prime}$ (resp. $U<U^{\prime}$ and $V \geq V^{\prime}$ ), then equation (3) is satisfied by setting $\mathfrak{z} m+1=-\mathfrak{z}_{m}$ and $Z_{m+1}=V^{\prime}-U+B_{3}+B_{4}-Z_{m}$ (resp. $\left.Z_{m+1}=V-U^{\prime}+B_{3}+B_{4}-Z_{m}\right)$ (see equation (12)). Similarly there exists $\left(\mathfrak{y}_{m+1}, Y_{m+1}\right)$ such that equation (4) is satisfied while $\left(\mathfrak{y}_{m}, Y_{m}\right)$ and $\left(\mathfrak{z}_{m+1}, Z_{m+1}\right)$ are fixed.

We proceed it for $m=n_{o}, n_{o}+1, \ldots$, and a solution $\left(\mathfrak{y}_{n}, Y_{n}\right),\left(\mathfrak{z}_{n}, Z_{n}\right)\left(n>n_{o}\right)$ is obtained. By applying a similar procedure to equation (4) for $n=n_{o}-1$, equation (3) for $n=n_{o}-1$ 
and so on, a solution $\left(\mathfrak{y}_{n}, Y_{n}\right),\left(\mathfrak{z}_{n}, Z_{n}\right)\left(n<n_{o}\right)$ is obtained. Thus we have a solution $\left(Y_{n}, Z_{n}\right)$ $(n \in \mathbb{Z})$ which satisfies the condition $\left(\mathfrak{y}_{n_{o}}, Y_{n_{o}}\right)=\left(\tilde{\mathfrak{y}}_{o}, y_{o}\right)$ and $\left(\mathfrak{z}_{n_{o}}, Z_{n_{o}}\right)=\left(\tilde{\mathfrak{z}}_{o}, z_{o}\right)$.

The uniqueness of the solution is not satisfied for some cases. In the case $U=U^{\prime}$ or $V=V^{\prime}$ in the proof of the proposition, i.e. the case $Y_{m}=A_{3}, A_{4}, A_{1}+m Q$ or $A_{2}+m Q$, we do not have uniqueness of the solution. Similarly in the case $Z_{m+1}=B_{3}, B_{4}, B_{1}+m Q$ or $B_{2}+m Q$, we do not have uniqueness of the solution. On the other hand, in the case $\mathfrak{y}_{m}=\mathfrak{z}_{m}=-1$ for all $m$, i.e. the case essentially equivalent to the ultradiscrete Painlevé VI without parity variables introduced by Ormerod [7] (see also [13]), we have the uniqueness of the initial value problem (see equations (13), (16)).

\section{Ultradiscrete Riccati-type equation and ultradiscrete Painlevé VI equation}

We show that the ultradiscrete Riccati-type equation with parity variables (equations (7), (8)) satisfies the ultradiscrete Painlevé VI equation with parity variables (equations (3), (4)).

Lemma 1. If $\max \left(X_{1}, X_{2}\right)=\max \left(X_{3}, X_{4}\right)$ and $\max \left(W_{1}, W_{2}\right)=\max \left(W_{3}, W_{4}\right)$, then

$$
\begin{aligned}
& \max \left(X_{1}+W_{1}, X_{3}+W_{3}, X_{2}+W_{4}, X_{4}+W_{2}\right) \\
& \quad=\max \left(X_{2}+W_{2}, X_{4}+W_{4}, X_{1}+W_{3}, X_{3}+W_{1}\right) .
\end{aligned}
$$

This lemma can be shown by case-by-case analysis (see [13], e.g. if $X_{1} \geq X_{2}, X_{3} \geq X_{4}$, $W_{1} \leq W_{2}$ and $W_{3} \leq W_{4}$, then $X_{1}=X_{3}, W_{2}=W_{4}$ and l.h.s. of equation (19) $=\max \left(X_{1}+\right.$ $\left.W_{1}, X_{1}+W_{3}, X_{2}+W_{2}, X_{4}+W_{2}\right)=$ r.h.s. of equation (19)). Note that it is an ultradiscrete analogue of the identity $e^{X_{1}}-e^{X_{3}}=e^{X_{4}}-e^{X_{2}}, e^{W_{1}}-e^{W_{3}}=e^{W_{4}}-e^{W_{2}} \Longrightarrow e^{X_{1}+W_{1}}+e^{X_{3}+W_{3}}+$ $e^{X_{2}+W_{4}}+e^{X_{4}+W_{2}}=e^{X_{2}+W_{2}}+e^{X_{4}+W_{4}}+e^{X_{2}+W_{3}}+e^{X_{3}+W_{1}}$.

Theorem 1. If $A_{1}+B_{3}+Q=A_{3}+B_{1}$ and $A_{2}+B_{4}=A_{4}+B_{2}$, then any solutions of the ultradiscrete Riccati-type equation with parity variables (equations (7), (8)) satisfy the ultradiscrete Painlevé VI equation with parity variables (equations (3), (4)).

Proof. This theorem is shown by the lemma and case-by-case analysis.

We show equation (4) for every values of parity variables $\mathfrak{z}_{m+1}, \mathfrak{y}_{m}, \mathfrak{y}_{m+1}$ by applying equations (7), (8) and the lemma.

In the case $\mathfrak{z}_{m+1}=\mathfrak{y}_{m}=\mathfrak{y}_{m+1}=1$, we set $X_{1}=m Q+A_{2}+B_{4}, X_{2}=Y_{m}+Z_{m+1}$, $X_{3}=A_{4}+Z_{m+1}, X_{4}=B_{4}+Y_{m}, W_{1}=m Q+A_{3}+B_{1}, W_{2}=Y_{m+1}+Z_{m+1}, W_{3}=A_{3}+Z_{m+1}$, $W_{4}=B_{3}+Y_{m+1}$. Then equations (7), (8) are written as $\max \left(X_{1}, X_{2}\right)=\max \left(X_{3}, X_{4}\right)$ and $\max \left(W_{1}, W_{2}\right)=\max \left(W_{3}, W_{4}\right)$, i.e. equations (9), (10). By applying the lemma, we have

$$
\begin{aligned}
\max & {\left[\left(m Q+A_{2}+B_{4}\right)+\left(m Q+A_{3}+B_{1}\right),\left(A_{4}+Z_{m+1}\right)+\left(A_{3}+Z_{m+1}\right),\right.} \\
& \left.\left(Y_{m}+Z_{m+1}\right)+\left(B_{3}+Y_{m+1}\right),\left(B_{4}+Y_{m}\right)+\left(Y_{m+1}+Z_{m+1}\right)\right] \\
= & \max \left[\left(Y_{m}+Z_{m+1}\right)+\left(Y_{m+1}+Z_{m+1}\right),\left(B_{4}+Y_{m}\right)+\left(B_{3}+Y_{m+1}\right),\right. \\
& \left.\left(m Q+A_{2}+B_{4}\right)+\left(A_{3}+Z_{m+1}\right),\left(A_{4}+Z_{m+1}\right)+\left(m Q+A_{3}+B_{1}\right)\right],
\end{aligned}
$$

which is equivalent to equation (4) in the case $\mathfrak{z}_{m+1}=\mathfrak{y}_{m}=\mathfrak{y}_{m+1}=1$ (i.e. equation (15)) by using the relation $A_{2}+B_{4}=A_{4}+B_{2}$.

We can show similarly equation (4) in the cases $\left(\mathfrak{z}_{m+1}, \mathfrak{y}_{m}, \mathfrak{y}_{m+1}\right)=(1,1,-1),(1,-1,1)$, $(1,-1,-1)$ by applying the lemma.

In the case $\left(\mathfrak{z} m+1, \mathfrak{y}_{m}, \mathfrak{y}_{m+1}\right)=(-1,1,1)$, equation (4) is shown by using equation (14). 
In the cases $\left(\mathfrak{z}_{m+1}, \mathfrak{y}_{m}, \mathfrak{y}_{m+1}\right)=(-1,-1,1),(-1,1,-1),(-1,-1,-1)$, there is no solution to equations (7), (8), and the theorem holds true.

Similarly equation (3) is shown for every values of parity variables $\mathfrak{y}_{m+1}, \mathfrak{z} m, \mathfrak{z} m+1$ by applying equations (7), (8) and using the relation $A_{1}+B_{3}+Q=A_{3}+B_{1}$. For details see [13].

The ultradiscrete Riccati equation in this paper never appear in the case $\mathfrak{y}_{m}=\mathfrak{z}_{m}=-1$, i.e. the case without parity variable discussed in [7]. Therefore ultradiscretization with parity variable is essential to obtain the ultradiscrete Riccati equation.

\section{Solutions}

\subsection{Solutions of the ultradiscrete Riccati-type equation}

We directly investigate solutions of the ultradiscrete Riccati-type equation with parity variables (see equations $(7),(8)$ ), which are also solutions of the ultradiscrete Painlevé VI equation with parity variables (equations (3), (4)). Although it would be natural to investigate solutions of the ultradiscrete Riccati-type equation by introducing the ultradiscrete hypergeometric equation, we leave it to a future problem. In this subsection, we assume that $Q>0, A_{1}+B_{3}+Q=A_{3}+B_{1}$ and $A_{2}+B_{4}=A_{4}+B_{2}$. Set

$$
h=A_{3}+B_{1}-A_{2}-B_{4}, \quad h^{\prime}=A_{3}-A_{4}-B_{3}+B_{4} .
$$

To find solutions of the ultradiscrete Riccati-type equation with parity variables, we set an ansatz that $\mathfrak{y}_{m}=-1$ and $\mathfrak{z}_{m}=+1$. Then equations (7), (8) are written as

$$
\begin{aligned}
& \max \left(m Q+A_{2}, Y_{m}\right)+B_{4}=Z_{m+1}+\max \left(A_{4}, Y_{m}\right), \\
& \max \left((m+1) Q+A_{1}, Y_{m+1}\right)+B_{3}=Z_{m+1}+\max \left(A_{3}, Y_{m+1}\right) .
\end{aligned}
$$

We specify the maximum in each term by

$$
m Q+A_{2} \geq Y_{m}, \quad A_{4} \leq Y_{m}, \quad(m+1) Q+A_{1} \geq Y_{m+1}, \quad A_{3} \leq Y_{m+1} .
$$

Then we have $(m+1) Q+A_{1}+B_{3}=Z_{m+1}+Y_{m+1}, m Q+A_{2}+B_{4}=Z_{m+1}+Y_{m}$ and $Y_{m+1}-Y_{m}=$ $A_{3}+B_{1}-A_{2}-B_{4}=h$. Hence we obtain solutions $Y_{m}=h m+c, Z_{m+1}=(Q-h) m+A_{2}+$ $B_{4}-c$ where $c$ is a constant which satisfies inequalities (20). Thus we have four solutions of equations $(7),(8)$ which has a parameter $c$ or $c^{\prime}$ with conditions as follows:

$$
\begin{aligned}
& \left(\mathfrak{y}_{m}, Y_{m}\right)=(-1, h m+c), \quad\left(\mathfrak{z}_{m+1}, Z_{m+1}\right)=\left(+1,(Q-h) m+A_{2}+B_{4}-c\right), \\
& \text { condition for equation (7): } \quad h m \geq A_{4}-c, \quad(Q-h) m \geq c-A_{2} \text {, } \\
& \text { condition for equation (8): } \quad h(m+1) \geq A_{3}-c, \quad(Q-h)(m+1) \geq c-A_{1} \text {, } \\
& \left(\mathfrak{y}_{m}, Y_{m}\right)=\left(+1, h m+A_{2}+B_{4}-c\right), \quad\left(\mathfrak{z}_{m+1}, Z_{m+1}\right)=(-1,(Q-h) m+c), \\
& \text { condition for equation }(7): \quad(Q-h) m \geq B_{4}-c, \quad h m \geq c-B_{2} \text {, } \\
& \text { condition for equation (8): } \quad(Q-h) m \geq B_{3}-c, \quad h m \geq c-B_{1} \text {, } \\
& \left(\mathfrak{y}_{m}, Y_{m}\right)=\left(-1, h^{\prime} m+c^{\prime}\right), \quad\left(\mathfrak{z}_{m+1}, Z_{m+1}\right)=\left(+1, h^{\prime} m+c^{\prime}+B_{4}-A_{4}\right), \\
& \text { condition for equation (7): } \quad h^{\prime} m \leq A_{4}-c^{\prime}, \quad\left(Q-h^{\prime}\right) m \leq c^{\prime}-A_{2} \text {, } \\
& \text { condition for equation (8): } \quad h^{\prime}(m+1) \leq A_{3}-c^{\prime}, \quad\left(Q-h^{\prime}\right)(m+1) \leq c^{\prime}-A_{1} \text {, } \\
& \left(\mathfrak{y}_{m}, Y_{m}\right)=\left(+1, h^{\prime} m+c^{\prime}-B_{4}+A_{4}\right), \quad\left(\mathfrak{z}_{m+1}, Z_{m+1}\right)=\left(-1, h^{\prime} m+c^{\prime}\right), \\
& \text { condition for equation (7): } \quad h^{\prime} m \leq B_{4}-c^{\prime}, \quad\left(Q-h^{\prime}\right) m \leq c^{\prime}-B_{2} \text {, } \\
& \text { condition for equation (8): } \quad h^{\prime} m \leq B_{3}-c^{\prime}, \quad\left(Q-h^{\prime}\right) m \leq c^{\prime}-B_{1} \text {. }
\end{aligned}
$$


We also have solutions of equations (7), (8) which do not contain parameters. One of them is the following solution with the conditions:

$$
\begin{aligned}
& \left(\mathfrak{y}_{m+1}, Y_{m+1}\right)=\left(-1, A_{2}+B_{4}-B_{1}\right), \quad\left(\mathfrak{z}_{m+1}, Z_{m+1}\right)=\left(+1, m Q+B_{1}\right), \\
& A_{2}+B_{4} \leq A_{3}+B_{1}, \quad B_{1} \leq B_{2}, \quad m Q \geq \max \left(A_{2}+B_{4}-A_{1}-B_{1}, B_{4}-B_{1}\right) .
\end{aligned}
$$

Besides this type, we have four types of solutions for $m \gg 0$ in each case of the signs $\left(\mathfrak{y}_{m}, \mathfrak{z}_{m}\right)=$ $(-1,+1),(+1,-1)$ and $(+1,+1)$ respectively. We also have four types of solutions for $m \ll 0$ in each case of the signs $\left(\mathfrak{y}_{m}, \mathfrak{z}_{m}\right)=(-1,+1),(+1,-1)$ and $(+1,+1)$ respectively. One of the solutions is as follows:

$$
\begin{aligned}
& \left(\mathfrak{y}_{m+1}, Y_{m+1}\right)=\left(+1, A_{3}\right), \quad\left(\mathfrak{z}_{m+1}, Z_{m+1}\right)=\left(+1, B_{4}\right), \\
& A_{3} \geq A_{4}, \quad B_{3} \leq B_{4}, \quad m Q \leq \min \left(A_{3}-A_{2}, B_{4}-B_{1}\right) .
\end{aligned}
$$

We can construct global solutions of the ultradiscrete Riccati-type equation with parity variables by patching solutions for each region of the variable $m$ suitably. If there exists a value $c$ such that

$$
\max \left(A_{1}, A_{4}, A_{2}+B_{4}-B_{1}, A_{1}-B_{1}+B_{2}\right) \leq c \leq \min \left(A_{2}, A_{3}, A_{3}-B_{3}+B_{4}, A_{2}+B_{4}-B_{3}\right),
$$

(the conditions $0 \leq h \leq Q$ and $0 \leq h^{\prime} \leq Q$ are implied), we have a solution written as

$$
\begin{aligned}
& \left(\mathfrak{y}_{m}, Y_{m}\right)= \begin{cases}\left(-1, h^{\prime} m+c\right), & m \leq 0, \\
(-1, h m+c), & m \geq 1,\end{cases} \\
& \left(\mathfrak{z}_{m}, Z_{m}\right)= \begin{cases}\left(+1, h^{\prime} m+B_{3}-A_{3}+c\right), & m \leq 0, \\
\left(+1,(Q-h) m+A_{1}+B_{3}-c\right), & m \geq 1 .\end{cases}
\end{aligned}
$$

Let $m_{0}<0$. If $0 \leq h \leq Q, 0 \leq h^{\prime} \leq Q, B_{3} \leq B_{4} \leq B_{1} \leq B_{4}+Q, A_{3}+B_{1} \geq A_{4}+B_{4}$, $\max \left(A_{2}, A_{4}\right) \leq A_{3}$ and there exists a value $c^{\prime}$ such that

$$
A_{4} \leq h^{\prime} m_{0}+c^{\prime} \leq \min \left(A_{3}, A_{4}+h^{\prime}\right), \quad\left(Q-h^{\prime}\right) m_{0}+\max \left(A_{1}, A_{2}\right) \leq c^{\prime},
$$

then we have a solution written as

$$
\begin{aligned}
& \left(\mathfrak{y}_{m}, Y_{m}\right)= \begin{cases}\left(-1, h^{\prime} m+c^{\prime}\right), & m \leq m_{0}, \\
\left(+1, A_{3}\right), & m_{0}+1 \leq m \leq 0, \\
\left(-1, h m+A_{2}\right), & m \geq 1,\end{cases} \\
& \left(\mathfrak{z}_{m}, Z_{m}\right)= \begin{cases}\left(+1, h^{\prime} m+B_{3}-A_{3}+c^{\prime}\right), & m \leq m_{0}, \\
\left(+1, B_{4}\right), & m_{0}+1 \leq m \leq 1, \\
\left(+1,(Q-h)(m-1)+B_{4}\right), & m \geq 2 .\end{cases}
\end{aligned}
$$

Let $m_{0}>0$. If $0 \leq h \leq Q, 0 \leq h^{\prime} \leq Q, B_{4} \leq B_{1} \leq B_{2}, A_{1}+B_{1} \leq A_{2}+B_{4} \leq A_{1}+B_{1}+Q$, $A_{2} \leq \min \left(A_{1}, A_{3}\right)+Q, A_{1} \geq A_{4}, A_{2}+B_{3} \leq B_{4}+A_{3}+Q$ and there exists a value $c$ such that

$$
h\left(m_{0}-1\right)+B_{1} \leq c \leq h m_{0}+\min \left(B_{1}, B_{2}\right), \quad(Q-h) m_{0}+c \geq \max \left(B_{3}+(Q-h), B_{4}\right),
$$

then we have a solution written as

$$
\left(\mathfrak{y}_{m}, Y_{m}\right)= \begin{cases}\left(+1, h^{\prime} m+A_{1}-B_{1}+B_{2}\right), & m \leq-1, \\ \left(-1, A_{2}+B_{4}-B_{1}\right), & 0 \leq m \leq m_{0}-1, \\ \left(+1, h m+A_{2}+B_{4}-c\right), & m \geq m_{0}\end{cases}
$$




$$
\left(\mathfrak{z}_{m}, Z_{m}\right)= \begin{cases}\left(-1, h^{\prime} m+B_{2}-Q\right), & m \leq-1 \\ \left(+1, A_{2}+B_{4}-A_{1}-Q\right), & m=0, \\ \left(+1,(m-1) Q+B_{1}\right), & 1 \leq m \leq m_{0} \\ (-1,(Q-h)(m-1)+c), & m \geq m_{0}+1\end{cases}
$$

Note that we have other solutions which can be obtained similarly.

We now fix the parameters by $A_{1}=25, A_{2}=46, A_{3}=67, A_{4}=23, B_{1}=59, B_{2}=65$, $B_{3}=1, B_{4}=42, Q=100$. Then $B_{1}+A_{3}=A_{1}+B_{3}+Q, A_{4}+B_{2}=A_{2}+B_{4}, h=38$ and $h^{\prime}=85$. Equation (21) is a solution of the ultradiscrete Riccati-type equation with parity variables, if $31 \leq c \leq 46$. Moreover equation (22) (resp. equation (23)) is a solution of the ultradiscrete Riccati-type equation with parity variables, if $m_{0}<0$ and $-85 m_{0}+23 \leq c^{\prime} \leq-85 m_{0}+67$ (resp. $m_{0}>0$ and $\left.21+38 m_{0} \leq c \leq 59+38 m_{0}\right)$. We also have other solutions. For example, if $m_{0}<0$ and $-85 m_{0}-18 \leq c^{\prime} \leq-85 m_{0}+23$, then we have the following solution:

$$
\begin{aligned}
& \left(\mathfrak{y}_{m}, Y_{m}\right)= \begin{cases}\left(-1,85 m+c^{\prime}\right), & m \leq m_{0}, \\
(+1,67), & m_{0}+1 \leq m \leq 0, \\
(-1,38 m+46), & m \geq 1,\end{cases} \\
& \left(\mathfrak{z}_{m}, Z_{m}\right)= \begin{cases}\left(+1,85 m-66+c^{\prime}\right), & m \leq m_{0}+1, \\
(+1,42), & m_{0}+2 \leq m \leq 1, \\
(+1,62 m-20), & m \geq 2 .\end{cases}
\end{aligned}
$$

If $m_{0}<0$ and $15 m_{0}+31 \leq c^{\prime} \leq 15 m_{0}+46$, then we have the following solution:

$$
\begin{aligned}
& \left(\mathfrak{y}_{m}, Y_{m}\right)= \begin{cases}\left(-1,85 m+c^{\prime}\right), & m \leq m_{0}, \\
(-1,100 m+31), & m_{0}+1 \leq m \leq 0, \\
(-1,38 m+31), & m \geq 1,\end{cases} \\
& \left(\mathfrak{z}_{m}, Z_{m}\right)= \begin{cases}\left(+1,85 m-66+c^{\prime}\right), & m \leq m_{0}, \\
(+1,100 m-35), & m_{0}+1 \leq m \leq 0, \\
(+1,62 m-5), & m \geq 1 .\end{cases}
\end{aligned}
$$

\subsection{Solutions of the ultradiscrete Painlevé VI equation without parity variables}

We now investigate solutions of ultradiscrete Painlevé VI with the fixed parity variable $\mathfrak{y}_{m}=$ $\mathfrak{z} m=-1$ for all $m$ (see equations (13), (16)).

We look for the solutions written as $Y_{m}=\delta m+\beta$ and $Z_{m}=\alpha m+\gamma$ for $m \gg 0$. We substitute them into equations (13), (16). Then we have $\alpha+\delta=Q, 2(\beta+\gamma)+\alpha=B_{3}+B_{4}+A_{1}+A_{2}$ and inequalities among the parameter. More precisely, if

$$
\begin{aligned}
& 0 \leq \alpha \leq Q, \quad 2(\beta+\gamma)+\alpha=B_{3}+B_{4}+A_{1}+A_{2}, \\
& \alpha(m+1)+\gamma \geq \max \left(B_{3}, B_{4}\right), \quad \alpha m+\min \left(A_{1}, A_{2}\right) \geq \beta, \\
& (Q-\alpha) m+\beta \geq \max \left(A_{3}, A_{4}\right), \quad(Q-\alpha) m+\min \left(B_{1}, B_{2}\right) \geq \alpha+\gamma,
\end{aligned}
$$

then the functions

$$
Y_{m}=(Q-\alpha) m+\beta, \quad Z_{m}=\alpha m+\gamma
$$

satisfy equations (13), (16). Note that the constraint condition $B_{3}+B_{4}+A_{1}+A_{2}=Q+A_{3}+$ $A_{4}+B_{1}+B_{2}$ is used to prove the equalities. Similarly, if

$$
0 \leq \alpha^{\prime} \leq Q, \quad \alpha^{\prime}+2\left(\gamma^{\prime}-\beta^{\prime}\right)=B_{3}+B_{4}-A_{3}-A_{4},
$$




$$
\begin{aligned}
& \alpha^{\prime}(m+1)+\gamma^{\prime} \leq \min \left(B_{3}, B_{4}\right), \quad \alpha^{\prime} m+\beta^{\prime} \leq \min \left(A_{3}, A_{4}\right), \\
& \left(Q-\alpha^{\prime}\right) m+\max \left(B_{1}, B_{2}\right) \leq \alpha^{\prime}+\gamma^{\prime}, \quad\left(Q-\alpha^{\prime}\right) m+\max \left(A_{1}, A_{2}\right) \leq \beta^{\prime},
\end{aligned}
$$

then the functions

$$
Y_{m}=\alpha^{\prime} m+\beta^{\prime}, \quad Z_{m}=\alpha^{\prime} m+\gamma^{\prime}
$$

satisfy equations (13), (16). We propose the following conjecture for solutions of ultradiscrete Painlevé VI without parity variables (equations (13), (16)), which is supported by several numerical solutions.

Conjecture 1. For every solution $Y_{m}, Z_{m}(m \in \mathbb{Z})$ of ultradiscrete Painlevé VI without parity variable (equations (13), (16)), there exist $m_{0}, m_{0}^{\prime} \in \mathbb{Z}$ and $\alpha, \beta, \gamma, \alpha^{\prime}, \beta^{\prime}, \gamma^{\prime} \in \mathbb{R}$ satisfying equations (24), (25) such that

$$
\begin{aligned}
& Y_{m}=\alpha^{\prime} m+\beta^{\prime}, \quad Z_{m}=\alpha^{\prime} m+\gamma^{\prime}, \quad m \leq m_{0}^{\prime}, \\
& Y_{m}=(Q-\alpha) m+\beta, \quad Z_{m}=\alpha m+\gamma, \quad m \geq m_{0} .
\end{aligned}
$$

We give examples of solutions. We consider solutions in the case $A_{1}=32, A_{2}=33, A_{3}=37$, $A_{4}=22, B_{1}=53, B_{2}=65, B_{3}=8, B_{4}=4$, and $Q=100$. We choose the initial values by $Y_{0}=43, Z_{0}=40$. Then the solution is written as

$$
Y_{m}=\left\{\begin{array}{ll}
95 m+111, & m \leq-1, \\
43, & m=0, \\
11 m+111, & m \geq 1,
\end{array} \quad Z_{m}= \begin{cases}95 m+40, & m \leq 0 \\
89 m-117, & m \geq 1\end{cases}\right.
$$

On different initial values $Y_{0}=43, Z_{0}=50$, the solution is

$$
Y_{m}=\left\{\begin{array}{ll}
85 m-81, & m \leq-8, \\
-669, & m=-7, \\
115 m+131, & -1 \leq m \leq-6, \\
43, & m=0, \\
-9 m+131, & 1 \leq m \leq 11, \\
9 m-72, & m \geq 12,
\end{array} \quad Z_{m}= \begin{cases}85 m-147, & m \leq-7, \\
115 m+50, & 0 \leq m \leq-6, \\
109 m-147, & 1 \leq m \leq 11, \\
1156, & m=12, \\
91 m+65, & m \geq 13 .\end{cases}\right.
$$

In these cases, the conjecture is true.

\section{Concluding remarks}

In Section 2, we obtained a ultradiscretization with parity variables of the $q$-difference Painlevé VI equation. A list of Painlevé-type equations of second order was obtained by Sakai [10], and some members in the list are $q$-difference Painlevé equations. We believe that ultradiscretization with parity variables of the $q$-difference Painlevé equations can be done.

Although we investigated solutions of ultradiscrete Riccati-type equation directly, we did not study ultradiscrete hypergeometric equation in this paper. A theory of ultradiscrete hypergeometric equations should be developed because it will have potential for applications to several equations including $q$-difference hypergeometric equations and ultradiscrete Painlevé equations.

A merit of ultradiscrete equations is that we may have exact solutions with the aid of computers. We formulated Conjecture 1 by calculating several solutions in use of a computer. On the other hand, Murata [5] obtained exact solutions with two parameters for a ultradiscrete Painlevé II equation. We hope to understand exact solutions for ultradiscrete Painlevé equations deeply. 


\section{Acknowledgments}

The authors would like to thank Professor Junkichi Satsuma for discussions and suggestions. They also thank the referees for valuable comments. The first author is partially supported by the Grant-in-Aid for Young Scientists (B) (No. 22740107) from the Japan Society for the Promotion of Science.

\section{References}

[1] Bruno A.D., Batkhin A.B. (Editors), Proceedings of the International Conference "Painlevé equations and related topics" (June, 2011, Saint Petersburg, Russia), De Gruyter Proceedings in Mathematics, De Gruyter, Berlin, 2012.

[2] Isojima S., Satsuma J., A class of special solutions for the ultradiscrete Painlevé II equation, SIGMA $\mathbf{7}$ (2011), 074, 9 pages, arXiv:1107.4416.

[3] Jimbo M., Sakai H., A q-analog of the sixth Painlevé equation, Lett. Math. Phys. 38 (1996), 145-154, chao-dyn/9507010.

[4] Mimura N., Isojima S., Murata M., Satsuma J., Singularity confinement test for ultradiscrete equations with parity variables, J. Phys. A: Math. Theor. 42 (2009), 315206, 7 pages.

[5] Murata M., Exact solutions with two parameters for an ultradiscrete Painlevé equation of type $A_{6}^{(1)}, S I G M A$ 7 (2011), 059, 15 pages, arXiv:1106.3384.

[6] Ohta Y., Ramani A., Grammaticos B., An affine Weyl group approach to the eight-parameter discrete Painlevé equation, J. Phys. A: Math. Gen. 34 (2001), 10523-10532.

[7] Ormerod C.M., Reductions of lattice mKdV to q-P $\mathrm{VI}_{\mathrm{V}}$, Phys. Lett. A 376 (2012), 2855-2859, arXiv:1112.2419.

[8] Ramani A., Grammaticos B., Hietarinta J., Discrete versions of the Painlevé equations, Phys. Rev. Lett. 67 (1991), 1829-1832.

[9] Sakai H., Casorati determinant solutions for the q-difference sixth Painlevé equation, Nonlinearity 11 (1998), 823-833.

[10] Sakai H., Rational surfaces associated with affine root systems and geometry of the Painlevé equations, Comm. Math. Phys. 220 (2001), 165-229.

[11] Takahashi D., Tokihiro T., Grammaticos B., Ohta Y., Ramani A., Constructing solutions to the ultradiscrete Painlevé equations, J. Phys. A: Math. Gen. 30 (1997), 7953-7966.

[12] Tokihiro T., Takahashi D., Matsukidaira J., Satsuma J., From soliton equations to integrable cellular automata through a limiting procedure, Phys. Rev. Lett. 76 (1996), 3247-3250.

[13] Tsutsui T., Ultradiscretization with parity variables of $q$-Painlevé VI, Master's Thesis, Chuo University, 2013 (in Japanese). 